\title{
Provisioning Remote Lab Support for IT Programs in Distance Education
}

\author{
Lakshmanan Senthilkumar \\ Department of Information Technology, \\ Sikkim Manipal University - Directorate of Distance Education, India \\ Email: senthil.k@smudde.edu.in,senthilkumarl@rediffmail.com
}

\begin{abstract}
In the recent past Internet has become the de-facto communication network. It is being prominently used by Telecommunication, Television and other such networks as a carrier network. The current Internet technology has matured enough to support both Non-Real Time and Real-Time Streaming applications. Recently, even the speed of the access network through which an end user accesses the Internet has also increased substantially. All these have given way to newer Applications for being ported on to the Internet. A similar attempt has been made here to extend the Networking Lab infrastructure to students who have enrolled for their higher education through Distance mode. These students who are spread across the country are able to access the Network Lab to perform their Lab Exercises Live on Network devices as part of their Practical Course.
\end{abstract}

Index Terms - Distance Education, Remote Laboratory, Networking Practical course, Internet Service, Assessment

\section{INTRODUCTION}

With the availability of larger bandwidth connections both at the core and at the access points, Internet has become a ubiquitous network. More recently it has found its way more prominently in to the education field. Irrespective of the level of education whether it is K-12 or higher, use of Internet as an education tool has become imminent. It is even more looked as an online thesaurus, encyclopaedia, and knowledge repository than more as a communication media for knowledge and information sharing.

In the Indian context especially when looking at its demography Internet will be an inevitable tool to provide education to all. India with a population of 1.21 billion people (2011 census) has $50 \%$ of it below the age of 25. More precisely, of the 1.21 billion, $13 \%$ of this is in the age group of $0-6$ years and overall 31\% of the total population is in the age group of $0-$ 14years of age.

For this future young India population huge investment in Education Infrastructure is required. As per Ministry of Human Resource Development (HRD) Annual Report 2010-11, India has 544 University level institutions, which includes 261 State Universities, 73 State Private Universities, 42 Central Universities, 130 Deemed Universities, 33 institutions of national importance and five institutions established under various State Legislations. In addition to this there are about 25951 Autonomous and Affiliated Colleges. This makes India having the largest education system in the world.

India's GER for the year 2010 is $12 \%$, much below the global average of $26 \%$ as on 2007.India with its large number of education institute is third largest in terms of enrolment, behind china and USA. However, India's GER is far lower than the developed and developing countries. The Indian government has set a target of achieving a 30\% GER by 2020 in the high education space. This would take the enrolment to around 40 million from the present 24 million. This in turn translates to an additional requirement of about 33000 institutions taking the investment from current Rs. 4620 million to about Rs. 2,3250million at an average growth rate of $18 \%$.

To achieve this target GER, an average of 10 institutions needs to be started per day for the next 9 years. This seems too unrealistic. Thereby use of Information communication Technology (ICT) is being sought of by the Indian government.As part of XI plan (2002 - 2007) the Indian Government has launched the 'National Mission on Education through ICT' to increase GER in higher education. As a policy for distance education updated as of August 2009, ICT has been made compulsory for all Higher Education Institutions imparting distance education.

As part of ICT initiative the National Programme on Technology Enhanced Learning (NPTEL) was conceived in 1999 to provide E-learning through online web and video courses in science and Engineering stream. The mission of NPTEL is to enhance the reach of quality Engineering education to a large section of students. As on 2010, 260 courses in technical 
education space has been developed and launched. As part of phase -2 of the project, an additional 950 new courses across 15 disciplines are being planned for development by 2012.

With the growing population who would be eligible for higher education, ICT is being seen as a tool to increase the GER and also to provide quality education. In the recent times Indira Gandhi national Open University (IGNOU) has started many e-initiatives such as eGyanKosh - a National digital repository to store, index and share the digital learning resources developed by open and distance learning institutions in the country, Sakshat - a National mission on Education through ICT (NME-ICT) with an objective of setting up a 'One Stop Education Portal'. The Sakshat initiative is seen as a tool for bringing together best experts in the country in their respective fields and best available knowledge resources on the web in the public domain. It seeks to standardize curriculum and learning materials across the country and keep them in tune with the latest trends so that Indian learners do not lag behind their foreign counterpart.

Following these initiatives an attempt had been made to extend Real Time Computer Networking Laboratory facilities to students spread across the country taking admission to the IT program.

The following section - II addresses the related work in the same area, section - III highlights the Indian distance education scenario, demography and the use of Internet, section - IV discuss on the use of technology to provide access to remote users to a centralized laboratory infrastructure, section $-\mathrm{V}$ gives a snapshot of analysis done to understand the adaptability of a remote infrastructure by the students, section - VI describes its future scope. Section - VII provides a conclusion of the paper.

\section{RELATED WORK:}

In the literature, papers are available which highlights how a remote laboratory has been setup with the use of Internet and online tools for higher education space. In [1]Empirical evidences are provided to show that students equally prefer to use remote lab environments as that of using the conventional laboratory. In the paper it is even indicated that students prefer using remote lab when compared to a software simulation of the same environment. A general method of creating web-based remote laboratory experimentation is given in the paper by $\mathrm{S}$. H. Chen et.al. [2]. The paper focuses more on how to control the real instruments through Internet and conduct the experimentation remotely.The paper [3]show case, how a physical lab is transformed into a Virtual Environment for extending the same facility for distance education. The challenges highlighted are with respect to the student familiarity with the application, necessary software installation and also the troubleshooting.

The paper by Swapna Kumar[4] indicates that the use of Web2.0 technology as a teaching and learning tool is much appreciated by the undergraduate students. The paper by Federica Oradini and Gunter Saunders[5] illustrates the use of an online tutoring tool 'My west minister', in which the students are keen to see their tutor active in the system. This was a factor for the system not been actively used by the student community. The paper by NaumanSaeed et.al. [6] provides an insight through a survey that today's learners are flexible in stretching their learning styles and are able to accommodate varying instructional strategies which use the emerging web technologies. Further the learner's technology preferences are not limited to a particular tool.

\section{INTERNET AND THE INDIAN DEMOGRAPHY}

Internet was initially designed as a primitive communication system supporting half-duplex communication. The then popular services were E-mail, Gopher, and then came the WWW. The first implementation of WWW is known as Web 1.0. The underlying communication protocol of it is HTTP and the web page content design was using HTML. The major objective of WWW was to create a mechanism through which people and machines could communicate in an easy and interactive manner. Now with the advent of newer technology innovate services facilitating various forms of People to People and Machine to Machine are being deployed on the Internet. This has led to the next generation of web services code named as Web 2.0.

The Indian Internet Statistics [7] shows an increase in the active Internet user base. From a mere 25,000 users in 1998 it has increased to about 100+ million Users in 2010. The Internet download speed in India is 0.7 Mpbs (world average was registered is $1.9 \mathrm{mbps}$ )and the upload speed is $1.44 \mathrm{Mbps}$ (rank 144).

Of the 1.21 billion India's population, $8.4 \%$ of them are internet users, $2.1 \%$ use Facebook as the Social Networking platform [8]. Of this $50 \%$ on Indians are aged 25 or below. India is the 5th largest country for the social Networking giant Facebook having 24 million users, of which 9 million have joined in last 6 months and it will be the 2nd largest by 2012. It is also reported that about 20 million 
Indians look for online job openings every month. LinkedIn, an online Social Networking platform for professional has 10 million users from India alone. Further it is also reported that Indian firms will be hiring 35\% of their staff requirement from LinkedIn and similar websites.

As per [9] mobile Internet users are growing fast in India. The Indian demography looks something similar to this: $67 \%$ of India's population has a mobile phone (i.e.) 810 million mobile subscribers, growing by 20 million every month. This when compared to TV viewers which is only about 520 Million. The mobile Internet users in India are young, with 94 per cent of them aged between 13 and 34 years. The number of unique users of Opera Mini in the country shot up by 267.3\% between January 2010 and 2011, giving a strong hint that mobile Internet users are growing rapidly in India [8].

In India the internet-capable mobile handsets is bigger (250 million units) than the installed base of desktop computers (around 35 million). With the current rate of growth of smartphone users it is inevitable that the mobile web growth will overtake fixed web sooner rather than later. This will undoubtedly have a huge effect on the way Mobile Internet is consumed in India [10].

\section{SYSTEM ARCHITECTURE}

A centralized Laboratory for the practical course 'Computer Networking' has been setup. The three system architectures which are used in setting up the laboratory are shown in Fig.1, 4 and 5.

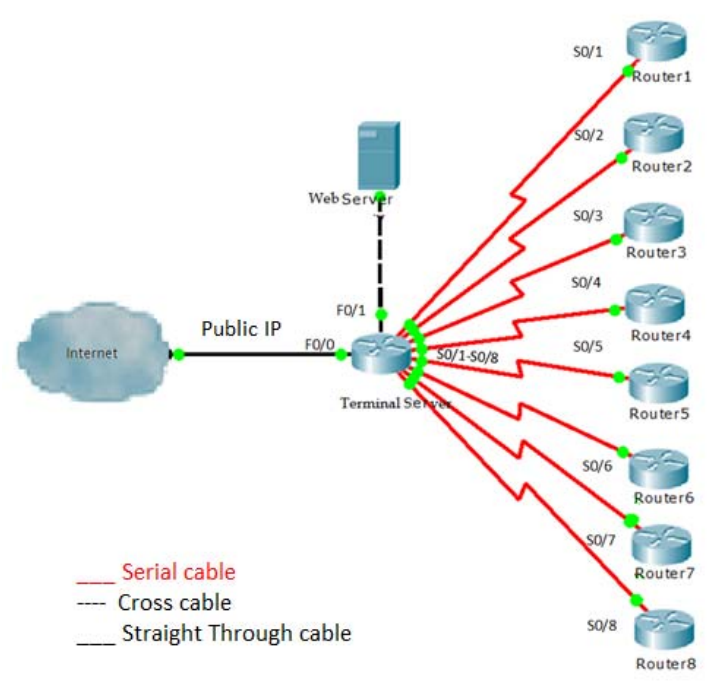

Figure 1.Architecture - 1: Centralized Network Laboratory- Having only routers
In Fig. 1, a POD(Pool of Devices) with a minimum one router is shown connected through a serial cable to a core router. Each POD is being identified through a physical port - id of the Terminal Server.

A web based GUI as shown in Fig. 2 is created through which a student can access the lab from a remote. A DNS to resolve the lab URL to the core router IP address is used. A student who wishes to access the lab needs to type the Lab URL in a web browser. At a given time one POD is assigned to a particular student. This mapping is done with the help of scripting at the web server, which again is connected to the core router (Fig. 1). Further all student detail validations, restrictions to access the day's assigned practical exercise is also again done through scripting at the web server end. To perform a particular lab exercise the student need to click on the title of the exercise. On clicking it, the student credentials are verified and a terminal window as shown in Fig.3 would appear, wherein the student would be then able to run the switch/ router commands to view, configure and also verify.

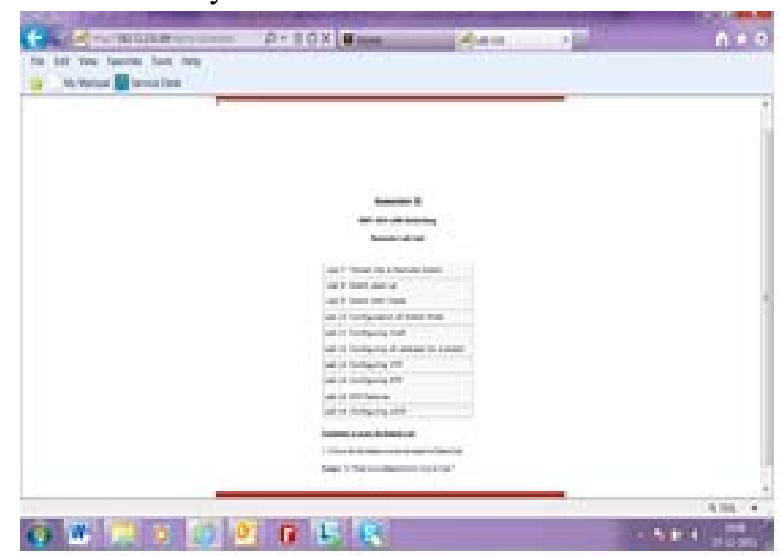

Figure 2. Web GUI to access the Remote Lab

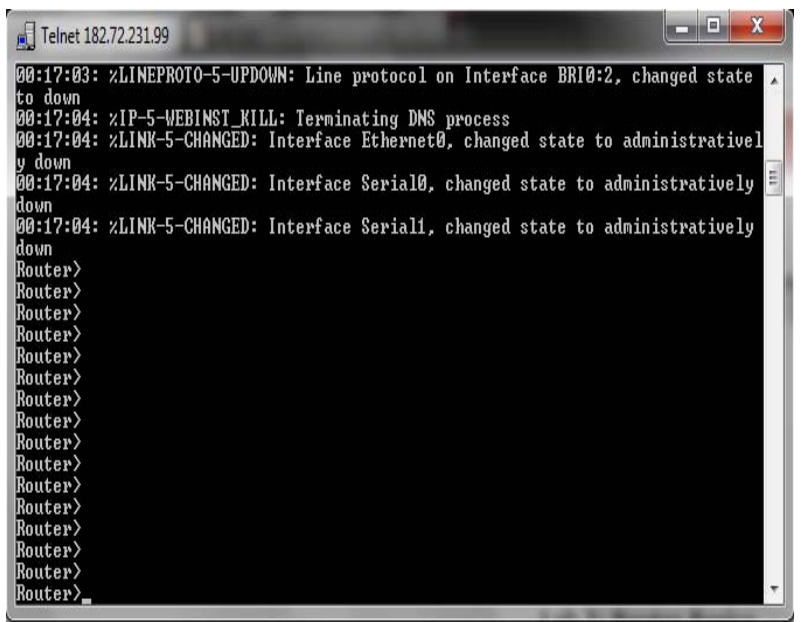

Figure 3. Terminal Window of a Switch 


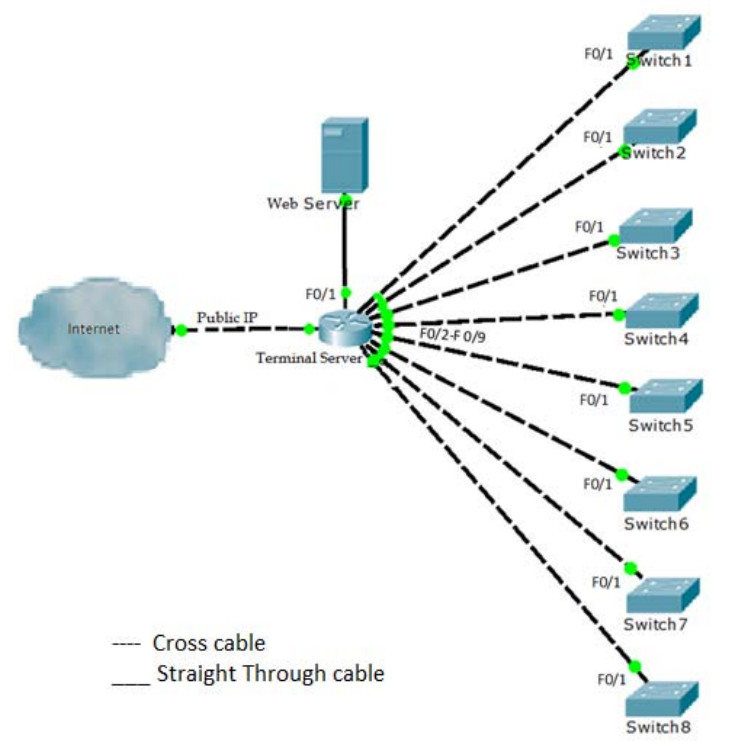

Figure 4.Architecture - 2: Centralized Network Laboratory - Having only switches

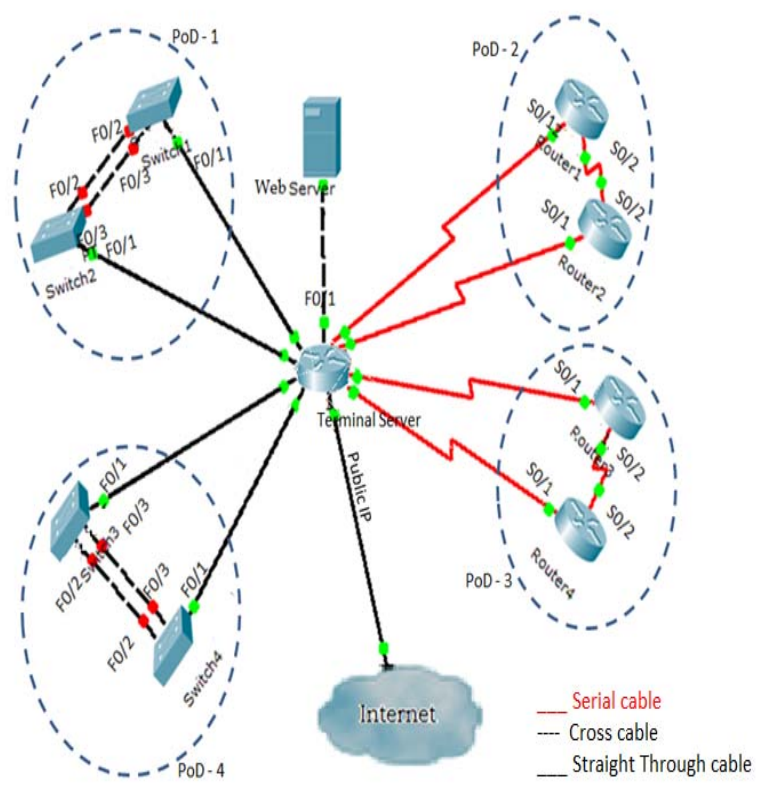

Figure5.Architecture - 3: Centralized Network Laboratory - Having both switch and routers

The other Lab architectures are as shown in Fig. 4 and 5. They are been setup when the Lab practical exercises are based only on routers and when on three devices (including the terminal server) in the POD for accessing respectively.

Fig. 4 highlights a Network Topology which is been setup for a practical course completely on switches. Again in this setup a POD comprise of only one device which is a switch. However, Fig. 5 show a Network topology which is been setup for a practical course comprising of both switches and routers. Here each POD consist of minimum two devices, both are either routers or switches. Devices within each POD are connected either using serial or cross cable, dependent on the device type. As shown in the Fig. PODs are connected with each other through a Terminal server.

The various Lab exercises which were performed by the students on a single switch includes: configuring port speeds, IP address, VLAN, Spanning Tree protocol, LACP protocol. The lab exercises performed on a single router device includes: configuring router interface speeds, IP address, subnet mask, different version of RIP protocol, OSPF, load balancing, access lists, VPN.

The Lab exercises which were performed on a POD having minimum of three devices (either all routers or switches) includes: configuration of port security, configuring protocols such as RSTP, MSTP, HSRP, VRRP, NTP and policy based routing.

\section{OBSERVATIONS}

The remote lab facility is being offered for the last six months for a chosen IT program offered under distance education. The Lab is being made available from morning 9:00am to 6:00pm. Each student had been signed two slots of 90 minutes in a week. This regular Lab session were evenly spread across different time slots. During this students were to practice and complete the practical exercises. A Lab utilization analysis was done to know the effectiveness of the resource and its acceptance in lieu of the physical resource available at the study centres. For this the first two batches (drives) of the students was used for the analysis (details Table - I). The student base is spread across the entire Indian geographical region with south and west of India having about $70 \%$ of the students enrolled for the program.

\section{TABLE I: NO. OF STUDENT ENROLLED FOR THE LAB FACILITY IN TWO DRIVES}

\begin{tabular}{|c|c|c|}
\hline Year & Drive & $\begin{array}{c}\text { Number of } \\
\text { students on roll }\end{array}$ \\
\hline 2011 & spring & 147 \\
\hline 2011 & Summer & 124 \\
\hline
\end{tabular}

A support service through Mentoring Chat session was run using WebEx Tool. The main objective of it is to conduct an online tutoring, clarify other doubts related to the practical exercise and also to clarify issues on access procedure to the remote lab. This support service of WebEx ILT (Instructor Led Training) was conducted once every week from 3:00pm 4:00pm.

The various metrics which were captured to study the utilization of the Remote Lab were 
i) Lab Utilization during Regular Practice Lab session

ii)Lab Utilization during University Practical session

iii) Lab Utilization across different time slots

iv)Participation in Mentoring Chat session

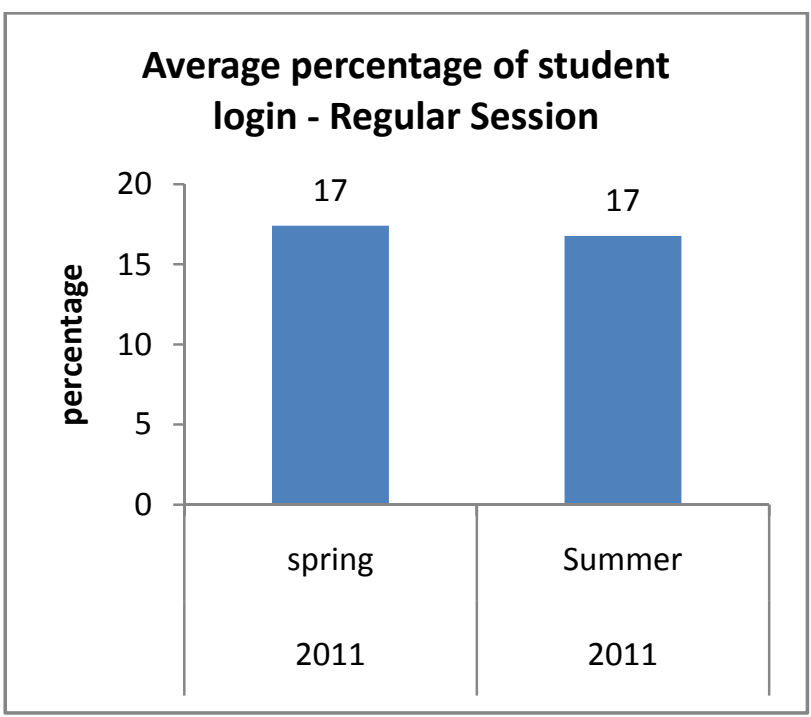

Figure 6.Lab Utilization during regular practice session

Fig. 6 shows the Lab utilization (average) by the students during their regular practice session slots. We found it at $17 \%$ when the drive was in progress. On interaction with the various learning centres and the students, the reasons found for such dismal lab utilizations were:

i) Few Learning centres which had their own lab infrastructure were not dependent on it.

ii) As per the distance education council (India) norms, for a 4 - credit course a maximum of only 12 contact sessions is necessary for a student to appear for the University Examination. Accordingly the monitored contact sessions is proportionate to the number credits of the course.

iii) Lack of Technical know-how to access the Remote facility.

iv) A large section of student being employed.

Also to understand this better, Lab utilization during the University Examination period was also studied.

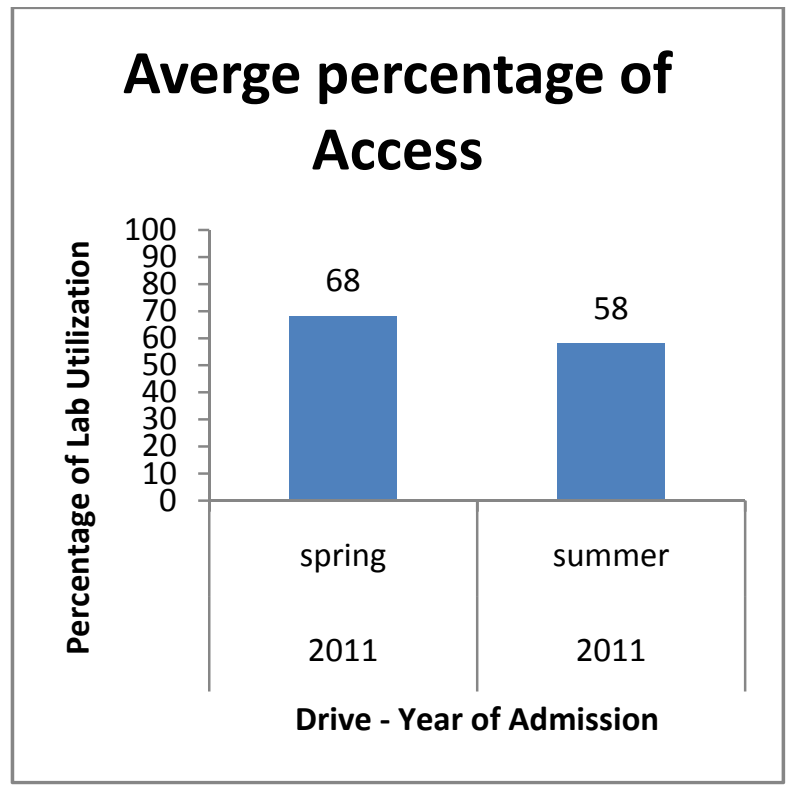

Figure 7. Lab Utilization (average) during the Term End University Examination session

We found the Lab utilization (average) at 68\% and 58\% during the two Term End University Examination drives are as shown in Fig. 7.Reason being, it is not mandatory to use this Lab infrastructure when the institute has its own setup. One another reason which is being attributedtowards it is power outages during the assigned time slots.

Another analysis was done to understand the time slot during which the Lab utilization has been at its peak. A slotted time structure is being used to assign the Lab resources to a student. Slot duration is of 90 minutes with a 15 minutes gap for the next slot. During this gap, Lab resources were reset to their initial state so that the next slot students can access the Lab to perform the practical exercises. With this arrangement a day has six slots, starting at 9:00 am to 7:15pm. The Lab schedules was prepared in such a manner that No Week Day/ Time Slot were fixed for a student (i.e.) the students could get different time slots at different days of the week. As shown in Fig. 8, the Lab Utilization was found at its peak during the second time slot which was from 10:45am to $12: 15 \mathrm{pm}$. 


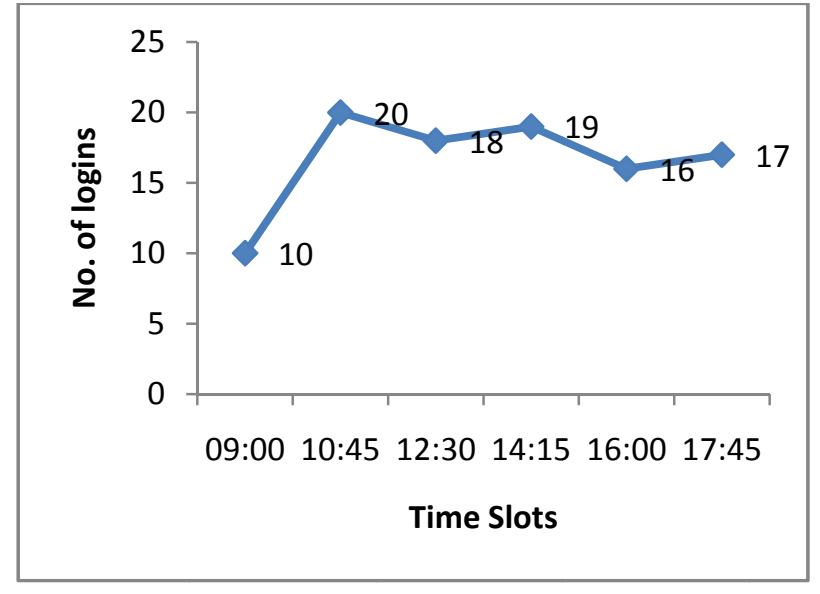

Figure 8.Peak Lab Utilization (average)-Time Slot

The other metric which was used to measure the online student engagement as part of the program delivery was the Student participation in the online Mentoring chat session.

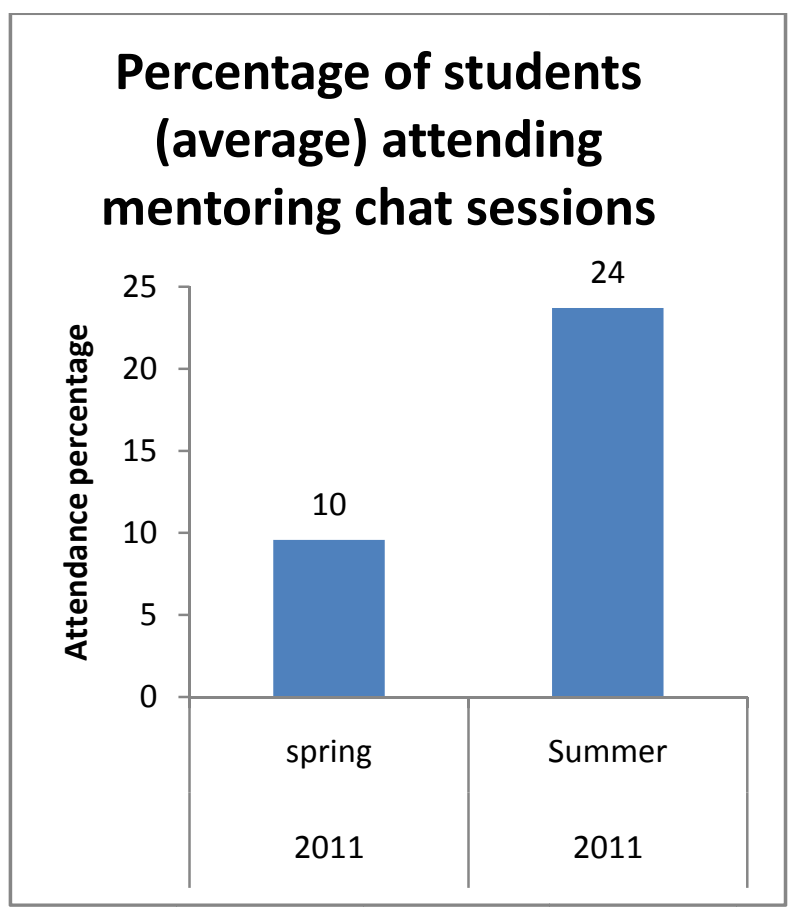

Figure 9.Mentoring Chat session -Attendance percentage

The mentoring chat session was run for duration of 120 minutes through WebEx tool. The participation was found at $10 \%$ for the first drive and $24 \%$ during the second drive. In the first drive student found difficult to use, as the media was new for them. However, in the subsequent drive as the learning centre faculty were tuned to use this media, the participation increased (fig. 9). This service was mainly used by students to clear their technical doubts on the practical component.

\section{FUTURE SCOPE}

1. To provide the same facility for the Post Graduate studentand see the Lab Utilization.

2. Encourage student to access the facility with incentives through better continuous assessment grading

3. To integrate social networking with the current mentoring chat session conducted through WebEx.

4. Organize T3 sessions at different locations, which could facilitate the study centre faculties adopt the facility with ease. They in turn could encourage students to use the centralized lab actively.

\section{CONCLUSION}

An attempt had been made to extend the reach of a Lab infrastructure to students admitted in to an undergraduate degree program offered through Distance mode. The student populationwhichis spread across the entire Indian geographical region has even helped us to reach some conclusions. One such fact finding is that the utilization of a lab facility, which could be increased only if the access is made mandatory. Also we could find that in certain regions especially where Internet connectivity is an issue the students prefer to complete their lab exercises through the Simulator.

\section{REFERENCES}

[1]BassemAlhalabi, M.K.Hamza, Ali Abu-El Humos, "Distance Education: Remote Labs Environment", ASEE Middle Atlantic Annual Meet proceedings,Spring 2008.

[2]S. H. Chen, R. Chen, V. Ramakrishnan, S. Y. Hu, Y. Zhuang, C. C. Ko and B. M. Chen, "Development of remote laboratory experimentation through Internet," Proceedings of the 1999 IEEE Hong Kong Symposium on Robotics and Control, Hong Kong, pp. 756-760, July 1999.

[3]StephenJ. Elliott and Eric P.Kukula, "The challenges associated with laboratory - based distance education”, EDUCASE Quarterly Magazine, Vol. 30 No. 1, 2007.

[4]Swapna Kumar, "Undergraduate Perceptions of the Usefulness of Web 2.0 in Higher Education: Survey Development”, Proceedings of 8th European Conference on E-learning, Italy,2009, pp 308 - 314 .

[5]FedericaOradini and Gunter Saunders, “The use of social Networking by students and staff in higher education”, iLearning Forum 2008, pp236-242

[6]NaumanSaeed, Yun Yang, SukuSinnappan, "Emerging web technologies in Higher Education: 
A case of incorporating Blogs, Podcasts and Social Bookmarks in a web programming course based on student's Learning style and Technology Preferences”, Journal of Educational Technology and Society, Vol. 12, Issue 4, 2009, pp. 98 - 109.

[7]Indian Internet Statistics http://www.onlinemarketingtrends.com/2011/01/indian-internet-statisticsfrom25000-to.html, January 21, 2011, Retrieved on March 20, 2012

[8]Vikas SN, "What does the Indian Social Media Landscape Look Like”? Web site: http://thenextweb.com/in/2011/06/24/whatdoes-the-indian-social-media-landscape-looklike/,2011,Retrieved March 20, 2012.

[9]Mobile Internet Users Growing Fast in India http://www.deccanherald.com/content/14664 0/mobile-internet-users-growing-fast.html, March 17, 2011,Retrievedon March 20, 2012

[10]Francis Tan, “India Part of the Next Web Family.”. Web site: http://thenextweb.com/in/2011/05/10/49of-indians-only-access-web-through-mobile/, 2011, Retrievedon March 20, 2012

Dr. Lakshmanan Senthilkumar was born in India in 1975. He received his Bachelor's degree in Computer Science \&Engineering from Annamalai University in 1996, Master's degree in the same branch from Madras University in 2000 and Ph.D. in Information and Communication Engineering from Anna University, Chennai in 2007. He is currently Professor and Head in the Department of Information Technology of Sikkim Manipal University - Directorate of Distance Education, India. His area of research includes provisioning QoS in Internet, Mobile Computing, and Application of IT in Higher Education space. 